\title{
Subunit dynamics in alpha-crystallin through deuteration-assisted small-angle neutron scattering
}

\author{
R. Inoue ${ }^{1}$, Y. Sakamaki', T. Takata ${ }^{1}$, K. Morishima ${ }^{1}$, K. Wood ${ }^{2}$, N. Sato ${ }^{1}$, A. Okuda ${ }^{1}$, M. Shimizu ${ }^{1}$, \\ R. Urade ${ }^{1}$, N. Fujii ${ }^{1}$, M. Sugiyama ${ }^{1}$ \\ ${ }^{1}$ Institute for Integrated Radiation and Nuclear Science, Kyoto University, Kumatori, Sennan-gun, Osaka 590-0494 Japan. \\ ${ }^{2}$ Australian Nuclear Science and Technology Organization, Lucas Heights, NSW Australia. \\ inoue.rintaro.5w@kyoto-u.ac.jp
}

Both the preservation of transparency and high refractive index is indispensable for the maintenance of normal function of eye lens. Especially, its high refractive index is attained by high protein concentration $(\sim 300 \mathrm{mg} / \mathrm{mL}$ in human eye lens). Since there exists no turnover in the eye lens, the eye lens is always at the risk of onset of aggregation. However, the long-term transparency in eye lens is preserved at least for several tens years. Then, how can eye lens maintain a long-term transparency? Key protein retarding the onset of abnormal aggregation is chaperone activity of alpha-crystallin, which exists as oligomers consisting of approximately 20 40 subunits of two homologues: alphaA-crystallin and alphaB-crystallin. Aiming at the elucidation of mechanism of its chaperone function, clarification of its quaternary structure has been challenged through crystallography techniques for long time. However, its quaternary structure has not been solved due to the availability of its crystal. To overcome such a situation, experimental trials revealing its quaternary structure have been tackled through state-of-the art experimental techniques. However, no consensus conclusions on such revealed structure have been drawn at present. We then reached one assumption that alpha-crsytallin intrinsically lacks robust quaternary structure (dynamic quaternary structure) to understand such diverse experimental results without inconsistency. It is also considered that such dynamic quaternary structure must be originated from subunit exchange between alpha-crsytallin oligomers.

To prove our expectation, we then try to apply deuteration assisted small-angle neutron scattering technique [1] for visualizing subunit exchange in alpha-crsytallin oligomer [2]. At the presentation, we will also discuss the effect of concentration on mechanism of subunit exchange in alpha-crsytallin oligomer [3].

[1] Sugiyama, M. et al. Biophys. J. (2011). 110, 2037.

[2] Inoue, R. et al. Sci. Rep. (2016). 6, 29208.

[3] Inoue, R. et al. Sci. Rep. (2021). 11, 2255.

Keywords: Small-angle neutron scattering; deuteration

Acta Cryst. (2021), A77, C780 